\title{
Fitoestrógenos y sus efectos sobre la Osteoporosis en la Mujer Posmenopáusica
}

\author{
Emilio González Jiménez ${ }^{a}$, Guillermo A. Cañadas de la Fuente ${ }^{a}$ \\ Rafael Fernández Castillo ${ }^{b}$, Judit Álvarez Ferre ${ }^{c}$, Carolina González Antón ${ }^{d}$
}

\author{
a Departamento de Enfermería. \\ Escuela Universitaria de \\ Ciencias de la Salud. \\ Universidad de Granada.

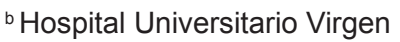 \\ de las Nieves de Granada. \\ Unidad de Hemodiálisis. \\ c Hospital Universitario \\ San Rafael. Unidad de \\ Hospitalización Crónica. \\ ¿Universidad de Granada. \\ Correspondencia: Emilio \\ González Jiménez, \\ Departamento de \\ Enfermería,Facultad \\ de Ciencias de la Salud, \\ Avda. de Madrid s/n, \\ 18071 - Granada, España. \\ Telf.: 667051570 , \\ correo electrónico: \\ emigoji@correo.ugr.es
}

Recibido el 28 de marzo de 2010.

Aceptado para su publicación el 6 de septiembre de 2010.

\begin{abstract}
RESUMEN
Los fitoestrógenos son sustancias de origen vegetal con efecto estrogénico débil, de inhibición enzimática y antioxidante. Desde un punto de vista clínico, se han barajado como una alternativa de tratamiento en la mujer menopáusica y sus síntomas asociados. Teniendo en cuenta que los estrógenos son los principales responsables del mantenimiento de la masa ósea en la mujer, inhibiendo la resorción ósea a través de su interacción con los osteoblastos, la reducción de sus niveles durante la menopausia constituirá un factor de riesgo para el desarrollo de osteoporosis en la mujer posmenopáusica. En este sentido, cabe plantearse si la terapia con fitoestrógenos constituye o no una herramienta terapéutica por su potencial efecto estrogénico en la prevención de la osteoporosis posmenopáusica. El objetivo de este trabajo ha sido proporcionar una revisión amplia y precisa acerca de la evidencia científica existente por el momento en torno al papel de los fitoestrógenos en la prevención de la osteoporosis en la mujer posmenopáusica y sus implicaciones en la clínica.
\end{abstract}

Palabras clave. Fitoestrógenos, Isoflavonas, Menopausia, Osteoporosis.

\section{ABSTRACT}

Phytoestrogens and their effects on Osteoporosis in Postmenopausal Women Phytoestrogens are plant substances with a weak estrogenic effect, an enzyme inhibitory effect and antioxidant activity. The possibility of using them for menopause associated symptoms has been entertained. Given that estrogens are primarily responsible for maintaining bone mass in women by inhibiting bone resorption through their interaction with osteoblasts, reducing estrogen levels during menopause constitutes a risk factor for developing osteoporosis in postmenopausal women. In view of this, the question arises as to whether phytoestrogens, due to their potential estrogenic effect, constitute or not a therapeutic tool in the prevention of postmenopausal osteoporosis. The aim of this study was to provide a comprehensive, accurate review on the available scientific evidence regarding the role of phytoestrogens in preventing osteoporosis in postmenopausal women and its implications in clinical practice

Key words. Phytoestrogens, Isoflavones, Menopause, Osteoporosis.

\section{FITOESTRÓGENOS Y SU RELEVANCIA CLÍNICA EN LA MUJER POS- MENOPÁUSICA}

Los fitoestrógenos son compuestos no esteró́dicos que derivan de diferentes especies vegetales. Se encuentran en plantas de la familia de las leguminosas, especialmente en las semillas de soja, pero también las contienen plantas de las familias de las solanáceas, gramíneas y rosáceas ${ }^{1}$. A pesar de que existen cuatro clases principales, nuestro interés se centrará en las isoflavonas, ya que en el momento actual son las que ofrecen mayores perspectivas terapéuticas. 
En el caso de la menopausia, el cese de la función ovárica va a conllevar toda una serie de consecuencias en salud para la mujer, apareciendo una compleja sintomatología que implica desde trastornos neurovegetativos hasta problemas osteoarticulares, circunstancia esta última que se complica si tenemos en cuenta la pérdida de masa ósea que tiene lugar como resultado de la deprivación ovárica y consiguiente déficit estrogénico ${ }^{2}$. Si además tenemos en cuenta el efecto protector de los estrógenos sobre la enfermedad coronaria, tenemos en la menopausia un factor de riesgo más para desarrollar trastornos cardiovasculares. Por todo lo expuesto, en la actualidad la terapia estrogénica también denominada terapia hormonal sustitutiva (THS) constituye uno de los pilares fundamentales en el tratamiento de la menopausia. Si bien y derivado de los beneficios hasta ahora reportados de esta terapia en pacientes posmenopáusicas, su utilización como alternativa natural a la terapia hormonal sustitutiva está cobrando una cada vez mayor relevancia clínica ${ }^{3}$. Ello en parte mediado por una mejor adhesión al tratamiento por parte del paciente dado su carácter de terapia "natural".

\section{ASPECTOS HISTÓRICOS DE LOS FITOESTRÓ- GENOS}

La historia natural de los fitoestrógenos como remedios terapéuticos es antigua. En 1931 Walz descubre una acción estrogénica en aquellos vegetales que contienen un elevado contenido en soja, dilucidando con ello la existencia de dos sustancias la genisteína y daidzeína, ambas pertenecientes al grupo de las isoflavonas.

Pero no será hasta 1946 cuando, asociado a sus efectos similares a los de los estrógenos, comiencen a valorarse los efectos de estas sustancias denominadas fitoestrógenos. Ahora bien, el interés científico por el análisis y estudio de los fitoestrógenos y sus efectos tendrá su origen en 1982 con Axelson y en 1984 con Setchell, considerándoseles a ambos responsables de la identificación de las isoflavonas en orina de humanos. A partir de ese gran descubrimiento en la década de los años 80 y hasta la actualidad serán numerosos los estudios epidemiológicos orientados a profundizar más sobre las potencialidades clínicas y terapéuticas de estas sustancias, centrándose en un gran número de casos en procesos relacionados con la mujer, concretamente con el climaterio.

\section{CARACTERÍSTICAS BIOQUÍMICAS Y MECA- NISMO DE ACCIÓN DE LOS FITOESTRÓGENOS}

Los fitoestrógenos, y en especial las isoflavonas, muestran una considerable semejanza en cuanto a su estructura química con los estrógenos. Se trata de moléculas que constan de varios grupos hidroxilos unidos a su vez a un grupo aromático ${ }^{7,8,9}$. En la actualidad se conocen hasta 230 tipos de isoflavonas diferentes entre sí, si bien no todas ellas van a cobrar igual relevancia en la clínica. Así, tres de los tipos más importantes son la daidzeína, también conocida como 7-dihidroxisoflavona, la genisteína, también denominada 5,7-trihidroxiflavona, y la gliciteína ${ }^{8,9}$.

En relación a su origen, los fitoestrógenos pueden tener directamente un origen vegetal o bien ser resultado del metabolismo de ciertos precursores presentes ya en las plantas. No debemos olvidar que en las plantas los fitoestrógenos intervienen regulando su crecimiento a la vez que las protege de los efectos dañinos de la radiación ultravioleta ${ }^{10}$.

Por su estructura, las isoflavonas se asemejan a los estrógenos, de ahí su relativa adhesión a los receptores estrogénicos tipo alfa y beta $(\alpha, \beta)$, aunque en mayor grado a los segundos ${ }^{11}$. Esta preferencia para ligarse con los receptores tipo $\beta$ llevará aparejada una mayor actividad de trascripción ${ }^{12}$. Esta circunstancia condiciona el mayor efecto de las isoflavonas en aquellos tejidos en que los receptores beta están presentes en cantidades relevantes, tales como el hueso, el sistema nervioso central y el endotelio vascular. No obstante, el mecanismo de acción de estas biomoléculas tras su adhesión a los receptores alfa y beta resulta controvertido, en tanto que se ha comprobado la posibilidad de que desarrollen no sólo efectos agonistas sino también de tipo antagónico ${ }^{12}$.

Del mismo modo, los fitoestrógenos, y de modo especial las isoflavonas, pueden ejercer sus efectos mediante procesos distintos a los ya mencionados, como por ejemplo a través de la inhibición o interferencia a nivel de numerosos procesos enzimáticos y de las propias enzimas, las cuales se encuentran implicadas en procesos de proliferación y diferenciación celular, agregación plaquetaria, actividad osteoclástica y metabolismo lipídico, entre otros. Algunas de las enzimas con esta capacidad son la 5-reductasa y 17 -hidroxidehidrogenasa entre otras $^{13}$. 


\section{BENEFICIOS CLÍNICOS DE LOS FITOESTRÓGE- NOS SOBRE LA OSTEOPOROSIS EN MUJERES POSMENOPÁUSICAS}

Entre los efectos orgánicos derivados de la menopausia uno de los más importantes por sus posibles consecuencias es la afectación ósea mediante osteoporosis. Con el cese de la función ovárica se reduce el efecto protector de los estrógenos sobre los huesos. Esta circunstancia se traduce en un desequilibrio entre el proceso de formación ósea y de reabsorción, fomentándose el efecto de éste último. El resultado de ello es la aparición y desarrollo en un gran número de pacientes con osteopenia y osteoporosis.

Diferentes estudios han mostrado los beneficios a este nivel de la terapia dirigida con un tipo de fitoestrógenos, las isoflavonas, ya que éstas incrementan los procesos de proliferación y diferenciación osteoblástica ${ }^{14}$. Ello parece poder explicarse por el efecto que las isoflavonas poseen sobre determinados receptores, como los beta, presentes en los osteoblastos ${ }^{15}$. Estudios con ratas tratadas con ovariectomía y sometidas a una dieta rica en soja han evidenciado una disminución de la pérdida de masa ósea, efecto que por otra parte ha resultado ser dosis dependiente en su mayoría de $\operatorname{casos}^{16,17}$.

De acuerdo con datos obtenidos a partir de ensayos clínicos en humanos donde se administraban cantidades predeterminadas de fitoestrógenos, y relacionándolos con diversos parámetros óseos, se han obtenido conclusiones alentadoras. De este modo en lo que respecta a parámetros bioquímicos de recambio óseo la práctica totalidad de los estudios hasta ahora realizados muestran una considerable reducción de las tasas de resorción ósea ${ }^{18}$, unido a una estimulación del proceso de formación ósea mediado por una elevación de los niveles de osteocalcina ${ }^{19}$. Centrándonos en el caso concreto de la genisteína, isoflavona presente en la soja y sus derivados, se ha visto cómo su consumo regular a través de la dieta mejora los parámetros de neoformación ósea a la par que reduce los indicadores de resorción ósea. Su mecanismo de acción no es del todo conocido, aunque estudios experimentales a partir de animales han revelado un efecto modulador del sistema de la osteoprotegerina y su sistema de ligandos. Por su parte, la osteoprotegerina se adhiere a sus ligandos regulando e inhibiendo con ello la diferenciación de osteoclastos y actividad de los mismos. La capacidad de la genisteína para estimular junto a los estrógenos la expresión de osteoprotegerina constituye el principio fundamental por el cual la genisteína altera el balance entre la osteoprotegerina y sus ligandos, mejorando con ello el recambio óseo.

Otros estudios ${ }^{19}$ han analizado la eficacia de una dieta rica en soja para la prevención de la osteoporosis posmenopáusica, comparándola con la terapia hormonal sustitutiva. Un estudio incluía 187 mujeres posmenopáusicas, aunque sin síntomas osteoporóticos. Se establecieron tres grupos para su comparación: uno con tratamiento hormonal, otro con dieta y un tercer grupo con placebo. Los resultados mostraron que la dieta no era efectiva como el tratamiento hormonal para disminuir el recambio óseo, sin embargo se pudo observar cómo ésta contribuía positivamente al estimulo de la actividad osteoblástica.

En lo que respecta a sus efectos sobre la densidad mineral ósea (DMO), diferentes estudios muestran una asociación estrecha entre consumo de isoflavonas a partir de vegetales y el incremento moderado de la densidad mineral ósea ${ }^{20,21}$. Chen y cols. en sus trabajos de 2003 y 2004 demostraron que los efectos de las isoflavonas presentes en la soja sobre la densidad mineral ósea son mayores en aquellas mujeres con poca o ninguna expresión estrogénica, en mujeres con menopausia tardía, bajo nivel de calcio en el organismo y aquellas con una masa ósea menor; es decir, en todas aquellas mujeres con un estatus menopáusico deteriorado. Otro estudio muestra que la suplementación diaria con 80 $\mathrm{mg}$ de isoflavonas mejora el contenido mineral óseo en determinadas localizaciones óseas como la cadera. Dicha mejora es atribuida al incremento de IGF-1, presuntamente causado por las isoflavonas y que favorece la actividad osteoblástica. A pesar de que la remodelación ósea es un proceso lento y sus efectos son a largo plazo, el estudio mostró efectos beneficiosos leves, pero significativos sobre el contenido mineral óseo ${ }^{23}$.

No obstante, parece prudente pensar en la necesidad de desarrollar más ensayos clínicos para poder verificar de forma más sólida la importancia y efectos de la terapia con fitoestrógenos sobre los procesos de pérdida ósea característicos de la mujer posmenopáusica. Si bien una dieta equilibrada como mecanismo de prevención debe constituir el primer escalón terapéutico en las pacientes con riesgo de osteoporosis, incluso la suplementación de esta dieta con soja puede presentar beneficios adicionales, sobre todo en grupos de alto riesgo ${ }^{24}$. 


\section{OTROS EFECTOS BENEFICIOSOS DE LOS FI- TOESTRÓGENOS EN LA MUJER POSMENO- PÁUSICA}

Otro de los síntomas que pueden verse aliviados mediante la terapia con fitoestrógenos (isoflavonas) en mujeres posmenopáusicas es la sintomatología vasomotora, con una tasa de incidencia próxima al $80 \%{ }^{25}$, si bien es cierto que el grado de percepción de su sintomatología va a variar dependiendo del ámbito poblacional y por ende del tipo de alimentación existente en esos territorios. Así, cabe destacar cómo la incidencia de esta sintomatología resulta mayor entre el colectivo de mujeres menopáusicas de nuestro país en comparación con mujeres de países asiáticos, en donde la ingesta de fitoestrógenos, concretamente de isoflavonas, a través de la dieta es mucho mayor. Los estudios aleatorizados disponibles muestran cómo dosis de isoflavonas de entre 34 a $100 \mathrm{mg} /$ día consiguen mejorías en dicha sintomatología, mejorías que se traducen en una reducción considerable de los sofocos, tan presentes en la mujer menopáusica ${ }^{26,27}$.

Otro síntoma a menudo mostrado por estas pacientes es la atrofia genital acompañada de una permanente sequedad vaginal, con la consiguiente distorsión que ello implica para conciliar la vida sexual con su pareja. Esta circunstancia comprende uno de los problemas más frecuentes y preocupantes en la mujer menopáusica. Pues bien, numerosos estudios has mostrado una mejoría de la sequedad vaginal a través de la terapia con isoflavonas ${ }^{27}$.

Por otra parte, los fitoestrógenos como agonistas estrogénicos van a carecer de efectos nocivos sobre el epitelio endometrial. Esto es, diferentes estudios ${ }^{28}$ han verificado la ausencia total de efectos proliferativos a nivel del endometrio con la consiguiente inexistencia de sangrados en las pacientes tratadas con fitoestrógenos ${ }^{29}$.

A nivel metabólico, las isoflavonas han demostrado una capacidad para inducir cambios a nivel de diferentes biomoléculas endógenas como la creatina y la creatinina, con el consiguiente efecto positivo a nivel de la función renal. Las isoflavonas, además, han demostrado un potencial efecto sobre procesos de diferenciación celular, evidenciando una capacidad para inducir la apoptosis en el caso de células cancerosas $^{30,31}$. Además, presentan propiedades antioxidantes y efectos inhibitorios de la agregación plaquetaria, al impedir la adhesión de moléculas como el tromboxano A2 a su receptor en las plaquetas, así como mejoras en el perfil lipídico ${ }^{32}$.

\section{CONCLUSIONES}

Basándose en las evidencias actuales, podemos concluir que los fitoestrógenos, y en especial las isoflavonas, constituyen una alternativa a la terapia hormonal sustitutiva en la mujer con menopausia, dados los numerosos beneficios derivados de su terapia en lo que concierne a los síntomas característicos de este período.

La existencia de datos significativos, como los reportados por Chiechi y cols. (2002) en favor de la terapia con isoflavonas como agentes de estimulo osteoblástico en la masa ósea de estas pacientes, determinan su consideración como una herramienta terapéutica más para el abordaje de la complicación más temida de la menopausia, la afectación ósea. Del mismo modo, la consecución de mejoras en el incremento de la densidad mineral ósea, en grado similar a la proporcionada por otras alternativas terapéuticas, determina el interés por seguir analizando y profundizando en sus efectos y mecanismos de actuación a éste nivel. Finalmente, la práctica inexistencia de efectos secundarios, tales como sangrados o trombos tan característicos de otras terapias, determina que el tratamiento con fitoestrógenos (isoflavonas) sea una herramienta no sólo beneficiosa para este tipo de pacientes, sino también segura, y quizás la mejor alternativa a la terapia convencional sintomática en la mujer posmenopáusica.

No obstante, resulta necesario seguir aunando criterios y conocimiento en torno a la biología molecular de estas sustancias y sus posibles efectos secundarios en las pacientes, pues no debemos olvidar que la mejor terapia no es aquella que mejor efecto muestra sobre la patología en sí, sino aquella que, además de lo anterior, menores efectos adversos proporciona al paciente.

\section{BIBLOGRAFIA}

1. Anderson JJ, Garner SC. Phytoestrogens and bone. Clin Endocrinol Metab. 1998; 12:543-57.

2. Umland EM, Cauffield JS, Kirk JK, Thomason TE. Phytoestrogens as therapeutic alternatives to traditional hormone replacement in postmenopausal women. Pharmacotherapy. 2000; 20:981-90.

3. Gass ML, Taylor MB. Alternatives for women through menopause. Am J Obstet Gynecol. 2001;185:47-56.

4. Walz E. Isoflavon-und Sapogenin-glucoside in Sojahispia. Justus Liebigs Ann Chem. 1931; 489:118-55.

5. Axelson M, Kirk DN, Farrant RD, Cooley G, Lawson AM, Setchel KDR. The identification of the weak oestrogen equol (7-hidroxi-3'(hidroxifenil) cromano) in human urine. Biochem J. 1982; 201:353-57. 
6. Setchell KDR, Borriello SP, Hulme P, Kirk DN, Axelson M. Non-steroidal oestrogens of dietary origin: possible roles in hormone dependent disease. Am J Clin Nutr. 1984; 40:56978.

7. Murkies AL, Lombard C, Strauss BJG, Wilcox G, Burger HG, Morton MS. Dietary flour supplementation decreases postmenopausal hot flushes: effect of soy and wheat. Maturitas. 1995; 21:189-95.

8. Dalais FS, Rice GE, Wahlqvist ML, Grehan M, Murkies AL, Medley G, et al. Effects of dietary phytoestrogens in postmenopausal women. Climacteric. 1998; 1:124-29.

9. Baber RJ, Templeman C, Morton T, Kelly GE, West L. Randomised placebo-controlled trial of an isoflavone supplement and menopausal symptoms in women. Climacteric. 1999; 2:85-92.

10. Boulet MJ, Oddens BJ, Lehert P, Verner HM, Visser A. Climacteric and menopause in seven south-east Asian countries. Maturitas. 1994; 19:157-76.

11. Kotsopoulos D, Dalais FS, Liang YL, McGrath BP, Teede $\mathrm{HJ}$. The effects of soy protein containing phytoestrogens on menopausal symptoms in postmenopausal women. Climacteric. 2000; 3:161-67.

12. Nikander E, Kilkkinen A, Metsa-Heikkila M, Adlercreutz $H$, Pietinen $\mathrm{P}$, Tiitinen $\mathrm{A}$, et al. A randomized placebo-controlled crossover trial with phytoestrogens in treatment of menopause in breast cancer patients. Obstet Gynecol. 2003; 101:1213-20.

13. Kuiper GG, Lemmen JG, Carlsson B, Corton JC, Safe SH, van der Saag PT, et al. Interaction of estrogenic chemicals and phytoestrogens with estrogen receptor beta. Endocrinology. 1998; 139:4252-63.

14. Choi EM, Suh KS, Kim YS, Choue RW, Koo SJ. Soybean ethanol extract increases the function of osteoblastic MC3T3-E1 cells. Phytochemistry. 2001; 56:733-39.

15. Rickard DJ, Monroe DG, Ruesink TJ, Khosla S, Riggs BL, Spelsberg TC. Phytoestrogen genistein acts as an estrogen agonist on human osteoblastic cells through esterogen receptors alpha and beta. J Cell Biochem. 2003; 89:633-46.

16. Arjmandi BH, Bimbaum R, Goyal NV, Getlinger MJ, Juma S, Alekel $\mathrm{L}$, et al. Bone-sparing effect of soy protein in ovarian hormone deficiency is related to its isoflavone content. Am J Clin Nutr. 1998; 68 suppl:1364-68.

17. Picherit C, Chanteranne B, Bennetau-Pelissero C, Davicco MJ, Lebecque P, Barlet JP, et al. Dose-dependent bonesparing effects of dietary isoflavones in the ovariectomized rat. Br J Nutr. 2001; 85:307-16.

18. Arjmandi BH, Khalil DA, Smith BJ, Lucas EA, Juma S, Payton $\mathrm{ME}$, et al. Soy protein has a greater effects on bone in postmenopausal women not on hormone replacement therapy, as evidenced by reducing bone resorption and urinary calcium excretion. J Clin Endocrinol Metab. 2003; 88:1048-54

19. Chiechi LM, Secreto G, D'Amore M, Fanelli M, Venturelli E, Cantatore $\mathrm{F}$, et al. Efficacy of a soy rich diet in preventing postmenopausal osteoporosis: the Menfis randomized trial. Maturitas. 2002; 42:295-300.
20. Kimira M, Arai $Y$, Shimoi K, et al. Japanese intake of flavonoids and isoflavonoids from foods. J Epidemiol. 1998; 8:168-75

21. Kritz-Silverstein D, Goodman-Gruen DL. Usual dietary isoflavone intake, bone mineral density, and bone metabolism in postmenopausal women. J Womens Health Gend Based Med. 2002; 11:69-78.

22. Chen YM, Ho SC, Lam SS, Ho SS, Woo JL. Beneficial effect of isoflavones on bone mineral content was modified by years since menopause, body weight, and calcium intake: a double-blind, randomized, controlled trial. Menopause. 2004; $11: 246-54$.

23. Chen YM, Ho SC, Lam SS, Ho SS, Woo JL. Soy isoflavones have a favorable effect on bone loss in Chinese postmenopausal women with lower bone mass: a double-blind, randomized, controlled trial. J Clin Endocr Metab. 2003; 88:4740-47.

24. Clifton-Bligh PB, Baber RJ, Fulcher GR, Nery ML, Moreton $T$. The effect of isoflavonas extracted from red clover (Rimostil) on lipid and bone metabolism. Menopause. 2001; 8:259-65.

25. Tomé D, Mariotti F. La soja en la alimentación. Alimentación, Nutrición y Salud. 2000; 7:31-3.

26. Rekers H. Mastering the menopause. En: Burger H, Boulet $M$, editors. A portrait of the menopause. Park Ridge, New Jersey: The Partenon Publishing Group, 1991. p. 23-43.

27. Nikander E, Kilkkinen A, Metsa-Heikkila M, Adlercreutz $H$, Pietinen $P$, Tiitinen A, et al. A randomized placebo-controlled crossover trial with phytoestrogens in treatment of menopause in breast cancer patients. Obstet Gynecol. 2003; 101:1213-20

28. Gilabert J, Abad A, Coloma F, Costa S, Payá V, Diago V, et al. Estudio prospectivo histeroscópico de la acción de las isoflavonas sobre el endometrio. Acta Gin. 2002; 49:21720.

29. Balk JL, Whiteside DA, Naus G, De Ferrari E, Roberts JM. A pilot study of the effects of phytoestrogen supplementation on postmenopausal endometrium. J Soc Gynecol Investig. 2002; 9:238-42.

30. Ingram D, Sanders K, Kolibaba M, et al. Case-control study of phyto-oestrogens and bresat cancer. The Lancet. 1997; 350:990-94.

31. Ishimi $\mathrm{Y}$, Miyaura C, Ohmura M, Onoe $\mathrm{Y}$, et al. Selective effets of genistein a soybean isoflavone, on B- lymphopoiesis and bone loss caused by estrogen deficiency. Endocrinology. 1999; 140 (4):1893-1900.

32. Dewell A, Hollenbeck CB, Brucen B. The effects of soyderived phytoestrogens on serum lipids and lipoproteins 217 isoflavonas en ginecología, terapia no convencional in moderately hypercholesterolemic postmenopausal women. J Clin Endocrinol Metab. 2002: 87:118-21. 